EPJ manuscript No.

(will be inserted by the editor)

\title{
Universal crossing probabilities and incipient spanning clusters in directed percolation
}

\author{
L. Turban ${ }^{\mathrm{a}}$ \\ Laboratoire de Physique des Matériaux ${ }^{\mathrm{b}}$, Université Henri Poincaré (Nancy I), BP 239, \\ 54506 Vandœuvre lès Nancy Cedex, France
}

Received 10 February 2003

\begin{abstract}
Shape-dependent universal crossing probabilities are studied, via Monte Carlo simulations, for bond and site directed percolation on the square lattice in the diagonal direction, at the percolation threshold. In a dynamical interpretation, the crossing probability is the probability that, on a system with size $L$, an epidemic spreading without immunization remains active at time $t$. Since the system is strongly anisotropic, the shape dependence in space-time enters through the effective aspect ratio $r_{\text {eff }}=c t / L^{z}$, where $c$ is a non-universal constant and $z$ the anisotropy exponent. A particular attention is paid to the influence of the initial state on the universal behaviour of the crossing probability. Using anisotropic finite-size scaling and generalizing a simple argument given by Aizenman for isotropic percolation, we also obtain the behaviour of the probability to find $n$ incipient spanning clusters on a finite system at time $t$. The numerical results are in good agreement with the conjecture.
\end{abstract}

PACS. 64.60.Ak Renormalization-group, fractal, and percolation studies of phase transitions - 05.50.+q Lattice theory and statistics (Ising, Potts, etc.) - 02.50.-r Probability theory, stochastic processes, and statistics

\section{Introduction}

Most of the properties of the critical state at a second order phase transition are related to the existence of self-similar correlated clusters. In the percolation problem, the critical point is characterized by the spontaneous occurence of infinite clusters of connected sites, crossing the system in the thermodynamic limit.

The study of crossing probabilities for standard percolation on finite systems at the percolation threshold has been an active field of research during the last decade [1-44] (for a recent review see [45]).

In two dimensions, the crossing probability $\pi$ can be defined as the probability to find at least one cluster joining two opposite edges of a rectangular-shaped system with length $L_{\|}$ and width $L_{\perp}$. At the percolation threshold, in the finite-size scaling (FSS) limit, i.e., when $L_{\|} \rightarrow \infty, L_{\perp} \rightarrow \infty$, while the aspect ratio $r=L_{\|} / L_{\perp}$ remains fixed, the crossing probability is a scale-invariant universal function $\pi(r)$ of the aspect ratio [12].

Following the extensive numerical study of Langlands $e t$ al. [1], an analytical expression for $\pi(r)$ at criticality was derived by Cardy [2]. Using the relation between percolation and the cluster formulation of the $q$-state Potts model in the limit $q \rightarrow 1$ [4647] as well as the techniques of boundary conformal field theory, he was able to calculate the probability for two non-overlapping segments on the edge of the half-plane to

\footnotetext{
a e-mail: turban@lpm.u-nancy.fr

b UMR CNRS 7556
}

be connected. The crossing probability in the rectangular geometry could then be deduced from the appropriate conformal mapping. One may notice that the scale invariance of $\pi(r)$ is a non-trivial property, resulting from the occurence of a vanishing scaling dimension $x(q)$ associated with a boundary condition changing operator of the Potts model in the limit $q \rightarrow 1$ for percolation.

Other exact crossing formulas were later obtained for different geometries [56]. Since then, mathematically rigorous proofs have been provided for some of these results [25 26].

Crossing probabilities on same-spin Ising clusters |27/28/29| and Potts clusters [30] in two dimensions have been also considered.

Recently, some attention has been paid to the properties of incipient spanning clusters [31-44]. The probability to find $n$ incipient spanning clusters, connecting two disjoint segments at the boundary of a finite critical system, has been studied through Monte Carlo simulations [31-39]. Rigourous bounds on the spanning probability [4041] have been obtained as well as analytical expressions through conformal and Coulomb-gas methods [42 43 44].

In two recent works [4849], we started a study of the critical crossing probability (in space-time) for off-equilibrium systems in 1+1 dimensions. In these strongly anisotropic systems, when the temperature-like scaling field $\Delta$ is non-vanishing, the correlation functions generally display an exponential decay, in the space and time directions, characterized by a correlation length $\xi$ and a relaxation time $\tau$ diverging respectively as [50]

$$
\xi=\hat{\xi} \Delta^{-\nu}, \quad \tau=\hat{\tau} \Delta^{-z \nu},
$$


when $\Delta \rightarrow 0$. The prefactors $\hat{\xi}$ and $\hat{\tau}$ are non-universal amplitudes and $z$ is the dynamical (anisotropy) exponent.

The directed percolation problem [5152 53] was studied numerically through Monte Carlo simulations in [48] and analytical results were obtained for an exactly solvable diffusionlimited coagulation process in [49. In these sytems, the crossing probability in the time direction $\pi_{t}$ is defined as the probability that the system of size $L$ remains active at time $t$. Anisotropic scaling [50 54] then implies that the appropriate aspect ratio is $r=t / L^{z}$, i.e., the rescaled time. We found that in the FSS limit, as for isotropic critical systems, the critical crossing probability is a scale-invariant universal function of an effective aspect ratio which is the product of $r$ by a non-universal constant $c$ [55].

In the present work, we continue the examination of the universal properties of the crossing probability $\pi_{t}$ in directed percolation which, in a dynamical interpretation, essentially corresponds to the survival probability of an epidemic spreading without immunization [56]. Section [2] is devoted to a detailed study of the influence of the initial state on the universal behaviour of $\pi_{t}$. In Section 3 we present some results concerning the scaling behaviour of the probability to find $n$ incipient spanning clusters, i.e., $n$ disjoint centres of infection surviving in the FSS limit.

\section{Critical crossing probability}

We study the critical crossing probability $P_{t}$, in the time direction, for bond and site directed percolation in $1+1$ dimensions through Monte Carlo simulations on the square lattice. The time axis is oriented in the diagonal direction. The space and time coordinates take alternatively integer and half-integer values on successive spatial rows. On a site located at $(x, t)$, with $1 \leq x \leq L$, two directed bonds are leaving, which terminate on the nearest-neighbour sites at $(x \pm 1 / 2, t+1 / 2)$ as shown in Figure 1 We use either free boundary conditions (FBC) or periodic boundary conditions (PBC) in the spatial direction. In the later case, $x$ is defined modulo $L$.

In the epidemic language, occupied (empty) sites correspond to infected (healthy) individuals. In the bond problem, the bonds are independently open with probability $p$. A site is occupied at $t+1 / 2$ when it is connected via an open bond to a site which was occupied at time $t$. In the site problem all the bonds are open and a site is occupied with probability $p$ at time $t+1 / 2$ when at least one of its first neighbours at time $t$ is occupied.

A directed percolation cluster is a collection of connected occupied sites starting from some source at $t=0$. We consider successively the three types of initial states shown in Figure 1 A sample contributes to the crossing probability when at least one cluster survives at time $t$. The simulations are performed at the percolation threshold, $p_{\mathrm{c}}^{\text {bond }}=0.644700185(5)$ for the bond problem and $p_{\mathrm{c}}^{\text {site }}=0.70548522(4)$ for the site problem. These values, as well as the dynamical exponent $z=$ 1.580745(10), are taken from [57]. The crossing probability $P_{t}(L, t, l)$ is determined as a function of the aspect ratio, $r=$ $t / L^{z}$, and the fraction $f=l / L$ of occupied sites in the initial state, using $10^{6}$ samples with sizes up to $L=2^{10}$. When $f$ is non-vanishing, $P_{t}$ converges to a scale-invariant function,
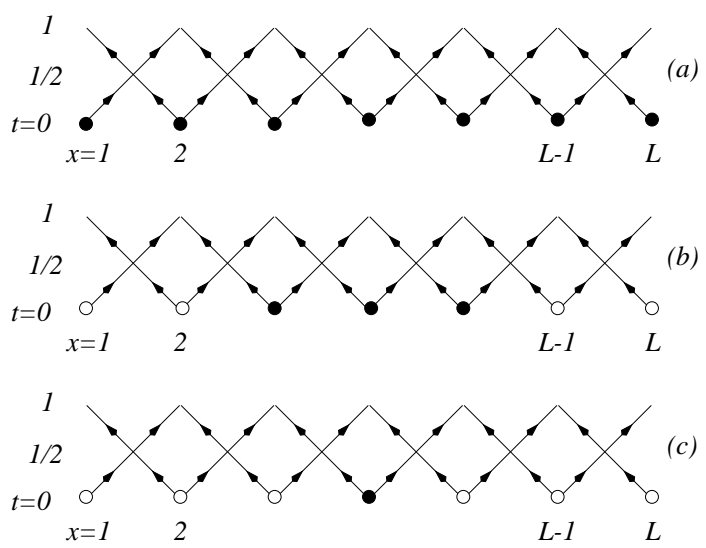

Fig. 1. Directed percolation on the square lattice in the diagonal direction. There are $L$ sites in the spatial direction which are either occupied $(\bullet)$ or empty $(\circ)$. Three types of initial states are considered with a) $L$ occupied sites, b) $l<L$ nearest-neighbour occupied sites and c) a single occupied site. In the last two cases, for FBC, the occupied sites are located in the middle of the system.

$\pi_{t}(r, f)$, in the FSS limit where $L, t$ and $l \rightarrow \infty$, while keeping $r$ and $f$ fixed.

\subsection{Initial state with a fully occupied lattice}

When the $L$ sites are randomly occupied with probability $p_{i}>$ 0 , the crossing probability converges to a scale-invariant function in the FSS limit. This is illustrated in Figure 2 in the case of site percolation with FBC. When $p_{i}=1$ the convergence is from above and the results obtained with $L=8$ are already quite close to the FSS limit. Thus $p_{i}$ is an irrelevant variable when $f>0$. In the following we always take $p_{i}=1$ in order to obtain a better convergence towards the scale-invariant behaviour.

We now consider site and bond directed percolation with either FBC or PBC in order to study the universality of the crossing probability. The lattice is fully occupied in the initial state. The behaviour of the crossing probability as a fonction of the aspect ratio is shown in Figure 3 The raw data converge quickly to scale-invariant functions as the system size is increased. They are shown in the inset for the largest size, $L=256$. Different functions are obtained for the two types of boundary conditions and the curves for the bond and site problems are shifted with respect to one another, along the horizontal axis, by an amount which is the same for FBC and PBC. The main figure shows the data collapse on universal curves, one for each type of boundary condition, after the results for site percolation have been shifted.

In order to explain this behaviour let us consider the scaling behaviour of the crossing probability for an off-critical system with a deviation $\Delta=\left|p-p_{c}\right|$ from the percolation threshold. Under an anisotropic change of the length scale by a factor $b$ the length transforms as $L^{\prime}=L / b$, the time as $t^{\prime}=t / b^{z}$ and $\Delta$ as $\Delta^{\prime}=b^{1 / \nu} \Delta$, where $\nu$ is the correlation length exponent defined in 1.1. Assuming that, as indicated by the Monte Carlo data, the crossing probability is scale invariant, then it satisfies 


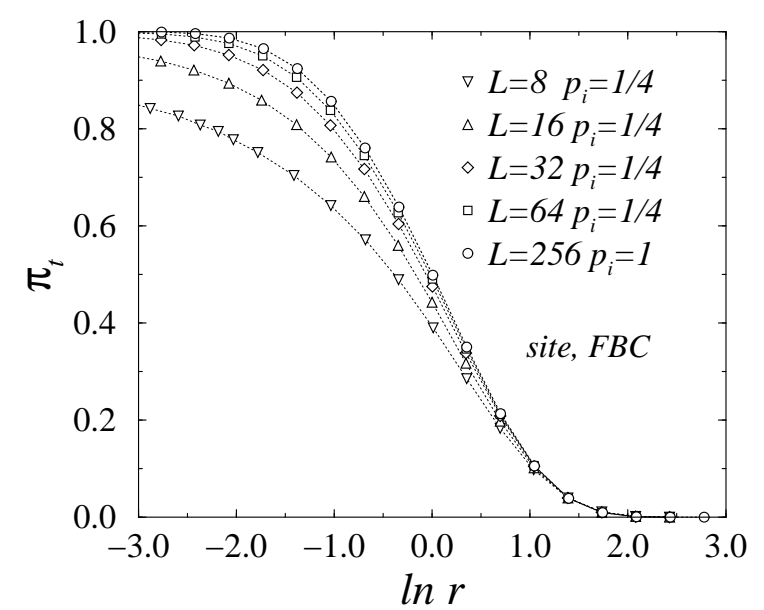

Fig. 2. Crossing probability as a function of $\ln r$ for site percolation with FBC. With increasing size, the values of $\pi_{t}$ obtained when the $L$ sites are randomly occupied in the initial state with probability $p_{i}=$ $1 / 4$, converge to the values obtained for $p_{i}=1$ on a lattice with size $L=256$. The statistical errors are smaller than the symbols.

the scaling relation

$$
P_{t}(L, t, \Delta)=P_{t}\left(\frac{L}{b}, \frac{t}{b^{z}}, b^{1 / \nu} \Delta\right) .
$$

With $b=\Delta^{-\nu}$ one obtains

$$
P_{t}(L, t, \Delta)=P_{t}\left(\frac{L}{\Delta^{-\nu}}, \frac{t}{\Delta^{-z \nu}}, 1\right)=g\left(\frac{L}{\xi}, \frac{t}{\tau}\right),
$$

where $g(u, v)$ is a universal fonction of its dimensionless arguments. The FSS limit corresponds to $\Delta=0$ and $b=L$ in 2.1., which gives

$$
P_{t}(L, t, 0)=P_{t}\left(1, \frac{t}{L^{z}}, 0\right)=\pi_{t}(c r)
$$

For each type of boundary conditions, the crossing probability is a scale-invariant universal function of the effective aspect ratio $r_{\text {eff }}=c r$. The non-universal amplitude $c$ depends on the choice of the length and time units. It can be expressed as a function of $\hat{\xi}$ and $\hat{\tau}$ [54]. Comparing (2.2) and (2.3), $r$ appears through the dimensionless ratio $v / u^{z}$ so that

$$
r_{\mathrm{eff}}=\frac{t / \tau}{(L / \xi)^{z}}=\frac{\hat{\xi}^{z}}{\hat{\tau}} r, \quad c=\frac{\hat{\xi}^{z}}{\hat{\tau}} .
$$

The non-universal constant $c$ is different for the two percolation problems. According to 2.3, for a given type of boundary condition, the crossing probabilities $\pi_{t}$ are identical for the site and bond problems when the values of the aspect ratio, $r_{\mathrm{s}}$ and $r_{\mathrm{b}}$, satisfy the relation $c_{\mathrm{s}} r_{\mathrm{s}}=c_{\mathrm{b}} r_{\mathrm{b}}$. Thus the data collapse in Figure 3 is obtained through a shift of the site percolation data by

$$
\delta_{\mathrm{sb}}^{r}=\ln r_{\mathrm{b}}-\ln r_{\mathrm{s}}=\ln \left(\frac{c_{\mathrm{s}}}{c_{\mathrm{b}}}\right)=.2476(8) .
$$

The numerical value of $\delta_{\mathrm{sb}}^{r}$ was estimated in [48] through a least-square fit.

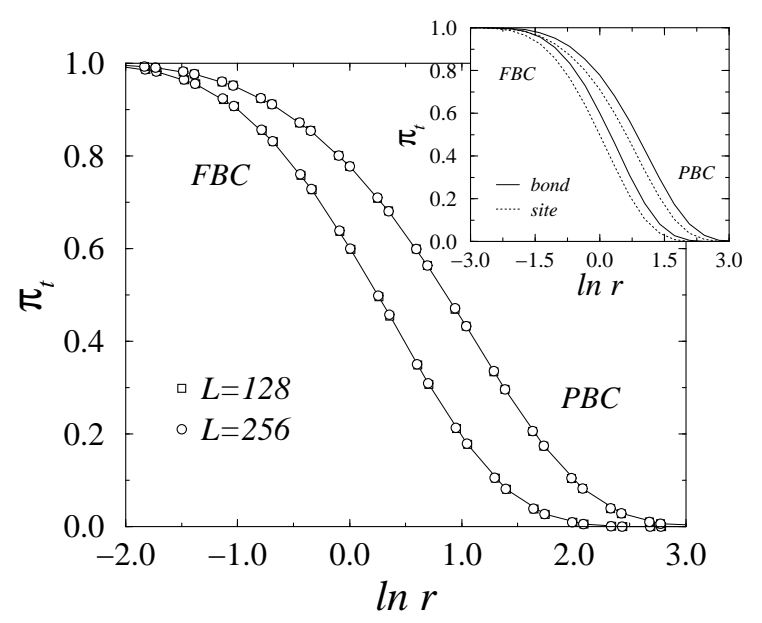

Fig. 3. Crossing probability as a function of $\ln r$ with a fully occupied lattice in the initial state. The Monte Carlo results for the largest size $(L=256)$ are shown in the inset for the bond and site problems with $\mathrm{FBC}$ and $\mathrm{PBC}$. The main figure shows the data collapse on two different universal curves for FBC and PBC in the scaling limit, after shifting the data for site percolation, as explained in the text. The statistical errors are smaller than the symbols.

The crossing probability can be calculated using a transfer matrix in the time direction $\mathrm{T}$, working with a restricted basis of states corresponding to configurations with at least one occupied site [58]. The matrix element $T_{\alpha \beta}$ gives the probability that the configuration in state $|\alpha\rangle$ at time $t$ leads to the configuration in state $|\beta\rangle$ at time $t+1$. Given an initial state $|i\rangle$, the crossing probability then reads

$$
\pi_{t}(c r)=\sum_{\alpha}\left\langle i\left|\mathbf{T}^{t}\right| \alpha\right\rangle .
$$

When $r=t / L^{z} \gg 1$, the leading contribution comes from the largest eigenvalue $\Lambda_{\max }<1$ of $\mathrm{T}$ and

$$
\pi_{t}(c r) \sim \Lambda_{\max }^{t}=\exp \left(-a \frac{c t}{L^{z}}\right)=\exp (-a c r)
$$

since the relaxation time $\left|\ln \left(\Lambda_{\max }\right)\right|^{-1}$ scales as $L^{z}$. In the argument of the exponential, $a$ is some universal constant. Thus $\ln \pi_{t}$ is a universal linear function of $c r$ when $r \gg 1$. This universal linear variation is shown in Figure 4 where $r$ for the site problem has been multiplied by $c_{\mathrm{s}} / c_{\mathrm{b}}=1.281(1)$ in order to eliminate the influence of the non-universal constant $c$ on the data collapse. Since the argument leading to (2.7) is quite general, the same behaviour is expected for the other types of initial states considered below.

\subsection{Initial state with a sequence of occupied sites}

Let us consider now the behaviour of the crossing probability when $l$ consecutive sites are occupied in the initial state, as shown in Figure $1 \mathrm{~b}$. The results for PBC and FBC are presented in Figures 5 and 6 respectively, for three values of the occupation ratio $f=l / L$, from top to bottom $f=1 / 2,1 / 4$, 


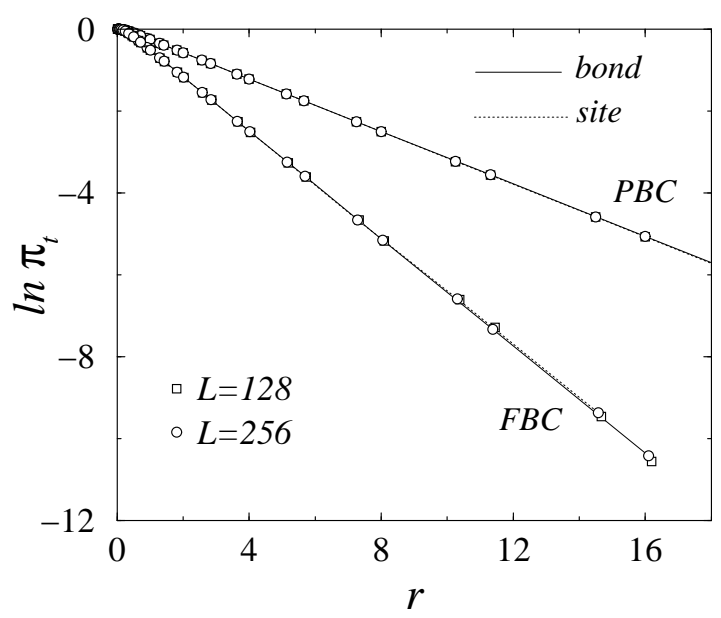

Fig. 4. Logarithm of the crossing probability as a function of $r$ with a fully occupied lattice in the initial state. For the site problem, $r$ has been multiplied by $c_{\mathrm{s}} / c_{\mathrm{b}}$. In the scaling limit, a linear universal behaviour is obtained, with different slopes for FBC and PBC.

$1 / 16$. The raw data for the largest size, $L=256$, are shown in the insets. As above, different curves are obtained for the site and bond problems. Shifting the site results by $\delta_{\mathrm{sb}}^{r}$, the data collapse on three universal curves, one for each value of $f$, as shown on the main figures.

The problem involves the new length scale, $l$, and the crossing probability scales as

$$
P_{t}(L, t, l, \Delta)=P_{t}\left(\frac{L}{b}, \frac{t}{b^{z}}, \frac{l}{b}, b^{1 / \nu} \Delta\right) .
$$

With $b=\Delta^{-\nu}$ one obtains

$$
P_{t}(L, t, l, \Delta)=P_{t}\left(\frac{L}{\Delta^{-\nu}}, \frac{t}{\Delta^{-z \nu}}, \frac{l}{\Delta^{-\nu}}, 1\right)=h\left(\frac{L}{\xi}, \frac{t}{\tau}, \frac{l}{\xi}\right),
$$

where $h(u, v, w)$ is a universal function of its arguments. In the FSS limit, at the critical point, equation (2.8) gives

$$
P_{t}(L, t, l, 0)=P_{t}\left(1, \frac{t}{L^{z}}, \frac{l}{L}, 0\right)=\pi_{t}(c r, f) .
$$

The dimensionless occupation ratio $f$ does not introduce any new non-universal constant in the scaling limit where $L$ and $l \rightarrow \infty$. Thus, as shown in Figures 5 and 6 the crossing probability is a scale-invariant universal function of the effective aspect ratio $r_{\text {eff }}$, which depends on the value of $f$ as well as on the boundary conditions. One may notice a slightly slower convergence to the limiting behaviour when $f$ decreases.

\subsection{Initial state with a single occupied site}

When a single site (located in the middle of the system for FBC) is occupied in the initial state, the crossing probability $\pi_{t}^{(1)}$ is no longer scale invariant. It acquires a dimension which is the scaling dimension of the order parameter,

$$
x_{m}=\frac{\beta}{\nu}=.252072(8),
$$

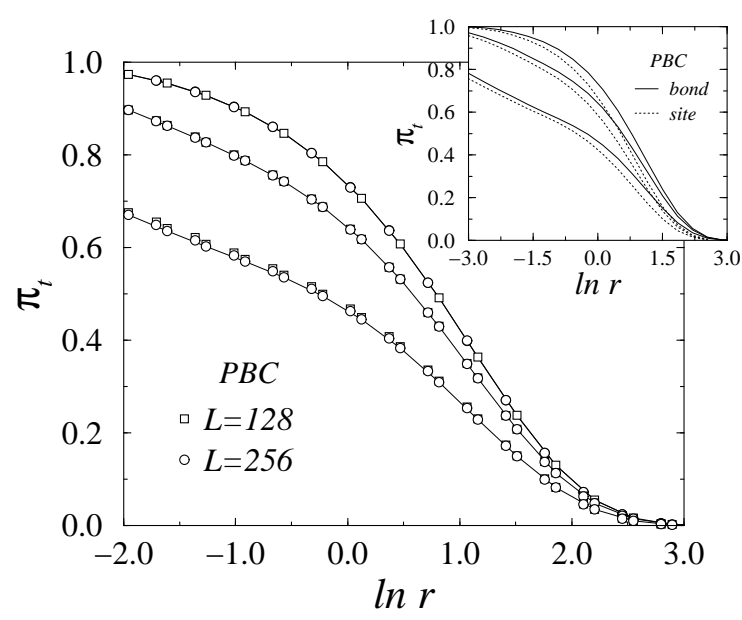

Fig. 5. Crossing probability as a function of $\ln r$ with a fraction $f=$ $l / L$ of occupied lattice in the initial state and PBC. The Monte Carlo results for the largest size $(L=256)$ are shown in the inset for the bond and site problems with $f=1 / 2,1 / 4$ and $1 / 16$ from top to bottom. The main figure shows the data collapse in the scaling limit on three different universal curves, one for each value of $f$, when the data for site percolation are appropriately shifted. The statistical errors are smaller than the symbols.

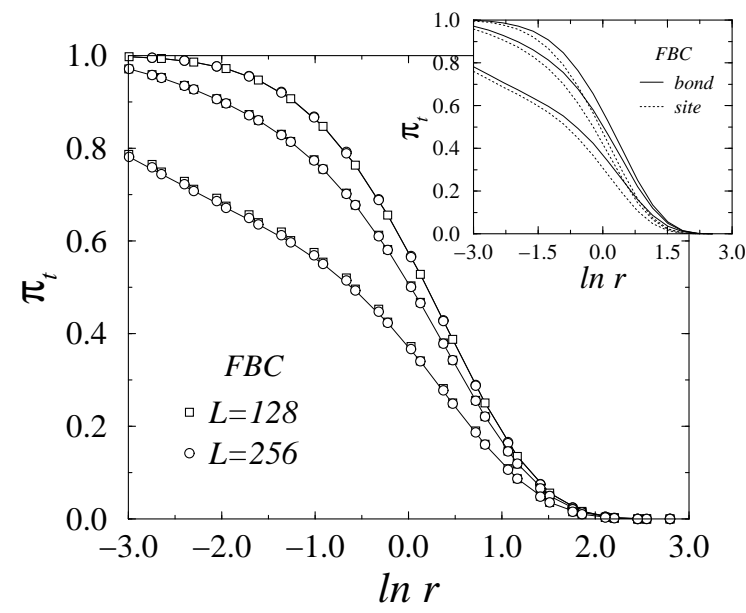

Fig. 6. As in Figure 5 for FBC.

corresponding to the values

$$
\beta=.276486(8), \quad \nu=1.096854(4),
$$

taken from [57]. This behaviour follows from the scaling of $P_{t}^{(1)}$, the probability that a cluster grown from a single central occupied site survives after $t$ time steps,

$$
P_{t}^{(1)}(L, t, \Delta)=b^{-x_{m}} P_{t}^{(1)}\left(\frac{L}{b}, \frac{t}{b^{z}}, b^{1 / \nu} \Delta\right) .
$$

On the infinite system with $b=t^{1 / z}$, one obtains

$$
P_{t}^{(1)}(\infty, t, \Delta)=t^{-x_{m} / z} P_{t}^{(1)}\left(\infty, 1, t^{1 / z \nu} \Delta\right)
$$

in agreement with equation (94) in reference [53], where the exponent $\beta^{\prime} / \nu_{\|}=\beta / \nu_{\|}=x_{m} / z$ for directed percolation with 


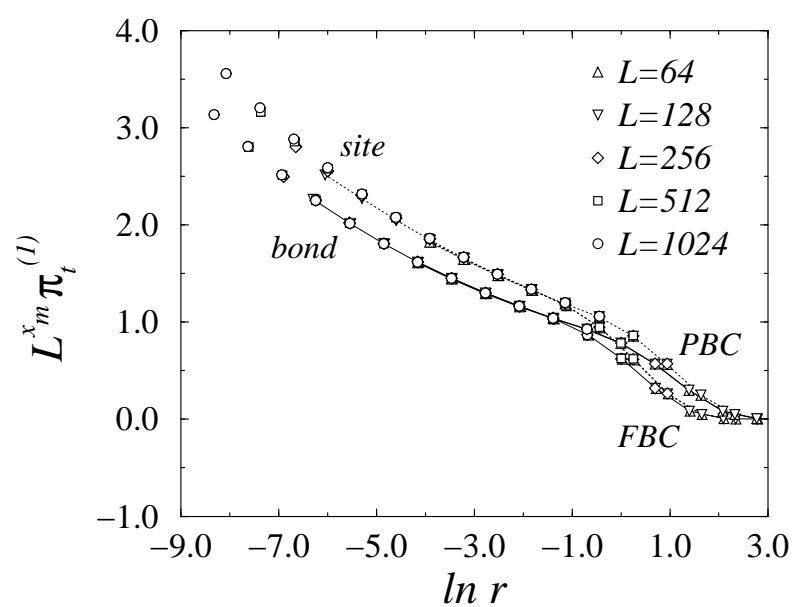

Fig. 7. Crossing probability for site (dotted line) and bond (full line) directed percolation with a single occupied site in the initial state as a function of $\ln r$. After rescaling $\pi_{t}^{(1)}$ by a factor $L^{x_{m}}$, the size effects are eliminated. Although the data for the site problem have been shifted by $\delta_{\mathrm{sb}}^{r}$, a different behaviour is still obtained for site and bond percolation.

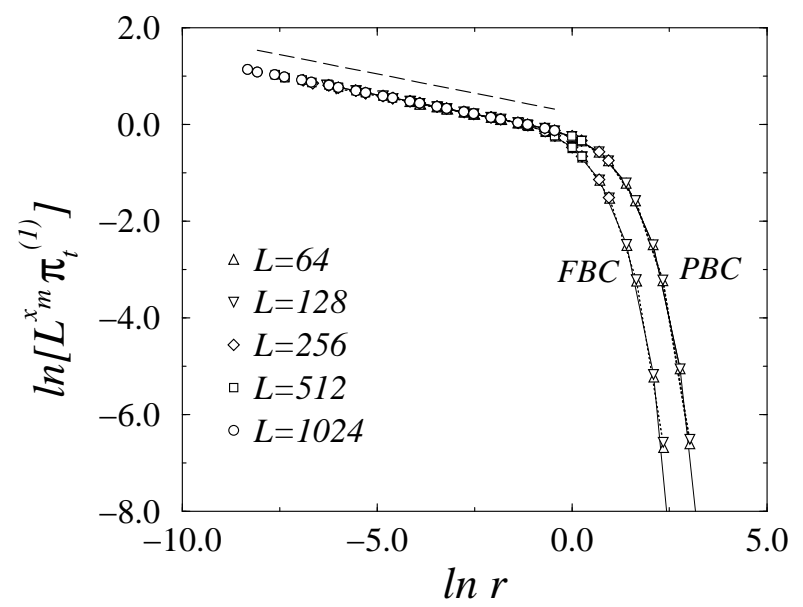

Fig. 8. Once $\ln \left(L^{x_{m}} \pi_{t}^{(1)}\right)$ for the site problem has been also shifted by $\delta_{\mathrm{sb}}^{\pi}$ in order to eliminate the influence of the non-universal amplitude in 2.19, the data collapse on two universal curves for FBC and PBC, respectively. For small values of $\ln r$ a linear variation is obtained with a slope $-x_{m} / z$, as indicated by the dashed line.

our notations. Taking $b=L$ at criticality $(\Delta=0)$ leads in the FSS limit to

$$
P_{t}^{(1)}(L, t, 0)=L^{-x_{m}} P_{t}^{(1)}\left(1, \frac{t}{L^{z}}, 0\right)=\pi_{t}^{(1)}(c r, L),
$$

As shown in Figure 7 a good data collapse is obtained for $L^{x_{m}} \pi_{t}^{(1)}$ with the different system sizes, $L=2^{6}$ to $2^{10}$. In this figure, the data for the site percolation problem have been shifted by $\delta_{\mathrm{sb}}$ given in (2.5) in order to take into account the non-universal constants $c_{\mathrm{s}}$ and $c_{\mathrm{b}}$.

For small values of $r, L^{z} \gg t$, the boundary conditions are irrelevant since, with a single occupied site in the initial state, the crossing cluster issued from the middle of the sample does not explore the boundary regions. For the same reason $\pi_{t}^{(1)}$ is independent of the size $L$ in this regime. Different curves are obtained for the site and bond problems for a given type of boundary conditions, meaning that a new non-universal constant is involved. In a log-log plot, it is clear that a single curve can be obtained through a vertical shift $\delta_{\mathrm{sb}}^{\pi}$ of the data for the site problem. The resulting data collapse is shown in Figure 8 Thus the new non-universal constant $A$ is a multiplicative one, either $A_{\mathrm{s}}$ or $A_{\mathrm{b}}$, and the shift is given by:

$$
\delta_{\mathrm{sb}}^{\pi}=\ln \left(\frac{A_{\mathrm{b}}}{A_{\mathrm{s}}}\right)=-.178(4) .
$$

It was calculated by first shifting the data of the site problem by $\delta_{\mathrm{sb}}^{r}$. Due to this horizontal shift of the site data, a direct calculation of the vertical shift is not possible. Thus, considering $y=\ln \left(L^{x_{m}} \pi_{t}^{(1)}\right)$ as a function $\ln r$, the data of the bond problem were fitted to a quadratic polynomial in the linear region, for small values of $\ln r$. Then $\delta_{\mathrm{sb}}^{\pi}$ was obtained as the average of $y_{\mathrm{b}}-y_{\mathrm{s}}$, evaluated at small enough values of $\ln r$ for the site problem.

Alternatively, the scaling behaviour of $\pi_{t}^{(1)}$ in 2.15 can be recovered by assuming that, asymptotically when $f \ll 1$, the scale-invariant crossing probability in $(2.10)$ behaves as

$$
\lim _{f \ll 1} \pi_{t}(c r, f)=f^{x_{m}} \varphi(c r)=\left(\frac{l}{L}\right)^{x_{m}} \varphi(c r),
$$

so that $L^{x_{m}} \pi_{t}^{(1)}=l^{x_{m}} \varphi(c r)$.

Furthemore the non-universal amplitude, $A_{\mathrm{s}}$ or $A_{\mathrm{b}}$, which appears only in $\pi_{t}^{(1)}$, i.e., when the number $l$ of occupied sites in the initial state is $\mathrm{O}(1)$, can be explained if one associates to these $l$ sites an effective length $l_{\text {eff }}$ given by

$$
l_{\mathrm{eff}}=l+\delta l, \quad \delta l=\delta l_{\mathrm{s}}, \delta l_{\mathrm{b}},
$$

where the increment $\delta l$ is $\mathrm{O}(1)$ and non-universal. Thus, replacing $f$ by $f_{\text {eff }}=(l+\delta l) / L$, there is no change in the FSS limit when $f>0$, but when $l=1$ we obtain

$$
L^{x_{m}} \pi_{t}^{(1)}=(1+\delta l)^{x_{m}} \varphi(c r)
$$

with a non-universal amplitude given by

$$
A=(1+\delta l)^{x_{m}} .
$$

According to 2.16) we have $\delta l_{\mathrm{s}}>\delta l_{\mathrm{b}}$.

Since, as noticed above, $\pi_{t}^{(1)}$ becomes independent of $L$ for small values of $r$, the scaling function $\varphi(w)$ with $w=$ $c r$ has to behave as $w^{-x_{m} / z}$ when $w \ll 1$. This leads to a slope $-x_{m} / z=-\beta / z \nu=-.159464(5)$ for the linear part of $\ln \left(L^{x_{m}} \pi_{t}^{(1)}\right)$ as a function of $\ln r$, in good agreement with the numerical results in Figure 8

\section{Critical spanning clusters}

In this Section we present a preliminary study of the scaling behaviour of the probability $\pi_{t n}(r)$ to find exactly $n$ independent 


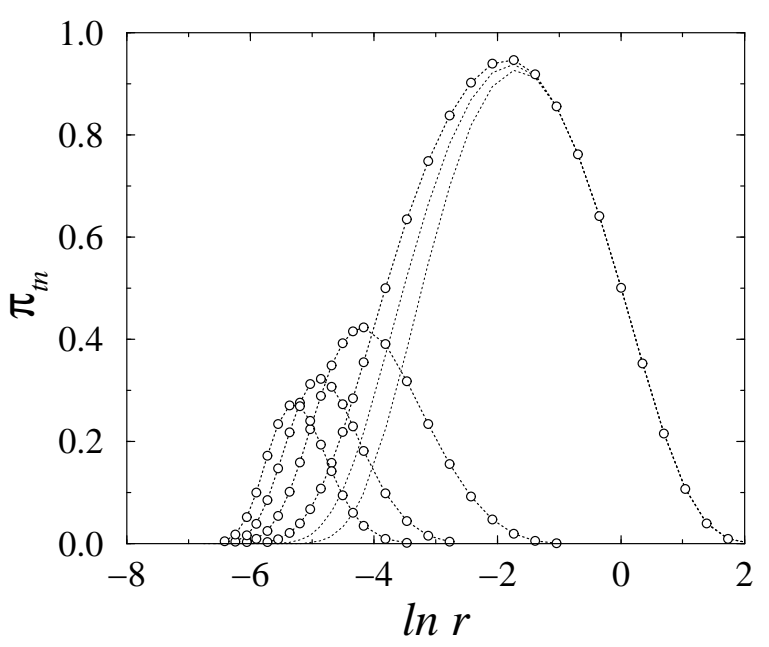

Fig. 9. Probability $\pi_{t n}$ to find $n$ critical spanning clusters in the time direction for the site problem with FBC when $L=256$. The number of clusters is $n=1$ to 4 from right to left. For $n=1$, the spanning probabilities obtained when $L=64$ and 128 are also shown.

clusters surviving after $t=r L^{z}$ time steps on a system with size $L$ in the FSS limit. In the epidemic interpretation $\pi_{t n}$ is the probability to find $n$ disjoint centres of infection surviving in the FSS limit. This problem presents some similarity with the statistics of family names first studied by Galton and Watson [59] (see also [6061]).

The spanning probability was estimated by generating $10^{6}$ samples with $L=64,128$ and 256 for the site problem with FBC or PBC and $L$ occupied sites in the initial state. As the size increases, it converges to a scale-invariant function $\pi_{t n}$ of the effective aspect ratio $r_{\text {eff }}$. A better convergence is obtained with FBC, thus we shall only analyse the results obtained with this type of boundary conditions. An example of the raw data for $\pi_{t n}$ at the largest sizes studied is shown in Figure 9

The scaling behaviour can be obtained by extending to the case of a strongly anisotropic system a simple argument proposed earlier for isotropic percolation [40 42].

Let $P_{t}(n, r)$ denote the probability to have exactly $n$ spanning clusters at time $t$, on a system in $d+1$ dimensions, with volume $L^{d}$. The scale invariance of $P_{t}$ implies that the size dependence is wholly contained in the aspect ratio $r$. Dividing the system in $b^{d}$ subsystems with the same volume $(L / b)^{d}$, as shown schematically in Figure 10 for $d=1$ and $b=n$, the dominant event will correspond to $n^{\prime}=n / b^{d}$ spanning clusters in space-time for each subsytem. Since the aspect ratio of the large system is $r=t / L^{z}$, it becomes $r^{\prime}=t /(L / b)^{z}=r b^{z}$ in the smaller subsystems. Thus, up to prefactors, one expects the following behaviour:

$$
P_{t}(n, r) \sim\left[P_{t}\left(b^{-d} n, b^{z} r\right)\right]^{b^{d}} .
$$

According to the transfer-matrix argument of Section 2.1 a leading exponential dependence on $t$ (through $r$ ) is expected. Thus, with $b=n^{1 / d}$, we obtain

$$
P_{t}(n, r) \sim\left[P_{t}\left(1, n^{z / d} r\right)\right]^{n} \sim \exp \left(-\alpha r n^{\omega}\right) .
$$

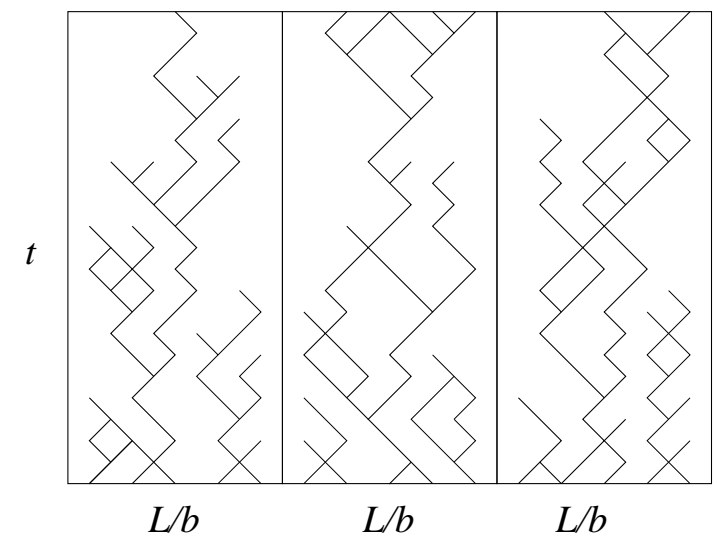

Fig. 10. $n$ spanning clusters on a system with size $L$ at time $t$ in $1+1$ dimensions are considered to result typically from the juxtaposition of $n / b$ spanning clusters on $b$ subsystems with size $L / b$.

Applying the first relation to the exponential form allows us to identify the exponent $\omega=1+z / d$ so that the scale-invariant crossing probability behaves as

$$
\pi_{t n}(r) \sim \exp \left(-\alpha r n^{1+z / d}\right),
$$

where $\alpha$ is a non-universal prefactor. Actually, the crossing probability $\pi_{t n}$, like $\pi_{t}$, is a universal function of the effective aspect ratio $\mathrm{cr}$. This scaling behaviour generalizes the form conjectured by Aizenman for isotropic percolation in $D=d+1$ dimensions [40], which was recently tested numerically with success [39]. The isotropic limit corresponds to $z=1$ and $d=D-1$ in 3.3 . The anisotropic result should remain valid below the upper critical dimension $d_{\mathrm{c}}=4$ for directed percolation [52]. At and above $d_{\mathrm{c}}$, as in the isotropic case, one expects a proliferation of spanning clusters, linked with the breakdown of hyperscaling [4062].

The Monte Carlo results in Figures 11 and 12 for the site and bond percolation problems with $\mathrm{FBC}$ at the largest size, $L=256$, are in good agreement with this scaling behaviour. The insets show the linear variation of $\ln \pi_{t n}$ when $r$ is sufficiently large. Different slopes are obtained for different values of $n$. As shown in the main figures, after multiplication by $n^{-(z+1)}$, a good data collapse is obtained.

The universality of $\pi_{t n}\left(r_{\mathrm{eff}}\right)$ is illustrated in Figure 12 The dotted line on the main figure corresponds to the site problem with $n=2$ after rescaling $r$ by the non-universal factor $c_{\mathrm{s}} / c_{\mathrm{b}}$. The remaining shift between the site and bond data is likely to be due to finite-size corrections.

\section{Conclusion}

For the directed percolation problem in the FSS limit, the crossing probability in the time direction, $\pi_{t}$, is a scale-invariant universal function of the effective aspect ratio $r_{\text {eff }}=c t / L^{z}$, appropriate for a strongly anisotropic system. It also depends on the fraction $f=l / L$ of occupied sites in the initial state, where $l$ is the length of the sequence of occupied sites. This function vanishes with $f$ as $f^{x_{m}}$, where $x_{m}=\beta / \nu$ is the scaling dimension of the order parameter. When the $L$ sites are occupied 


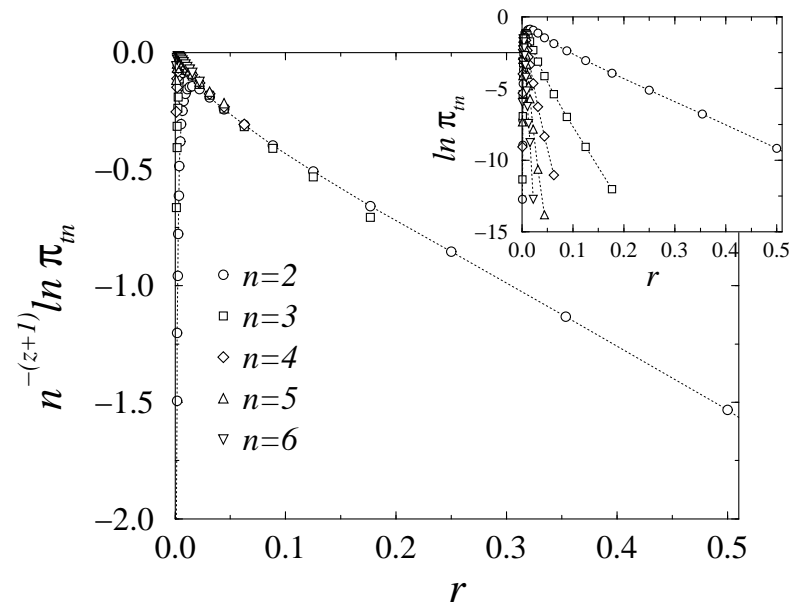

Fig. 11. Scaling behaviour of the crossing probability $\pi_{t n}(r)$ for the site percolation problem with FBC when $L=256$. The inset shows the linear variation of $\ln \pi_{t n}$ versus $r$ with a slope depending on $n=2$ to 6 from top to bottom. A good data collapse is obtained in the main figure, in agreement with 3.3 for $d=1$, when $\ln \pi_{t n}$ is rescaled by $n^{-(z+1)}$

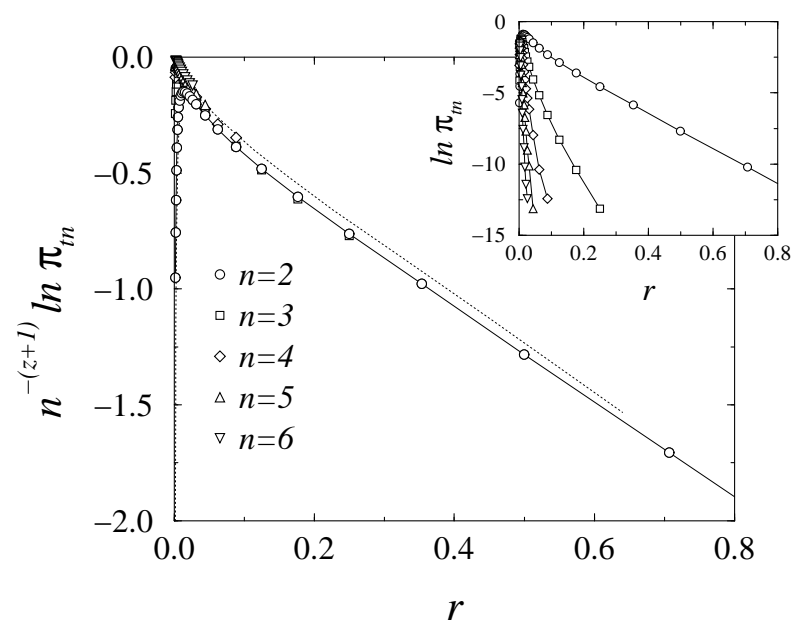

Fig. 12. As in Figure 11 for the bond percolation problem. The dotted line on the main figure corresponds to the site problem with $n=2$ with $r$ rescaled by the non-universal factor $c_{\mathrm{s}} / c_{\mathrm{b}}$.

with probability $p_{i}>0$ at $t=0$, the crossing probability is asymptotically the same as for a fully occupied system in the initial state.

Anisotropic scaling, together with the generalization of a simple geometrical argument due to Aizenman for isotropic percolation, leads to the scaling behaviour for the probability $\pi_{t n}$ to find $n$ critical directed percolation clusters surviving at time $t$ on a system with size $L^{d}$. The probability $\pi_{t n}$ is a scaleinvariant universal function which decays exponentially with $n^{1+z / d} r_{\text {eff }}$. The numerical data in $1+1$ dimensions support the conjectured expression although further work is needed with larger system sizes, in order to be closer to the true asymptotic behaviour. The scaling behaviour should be also examined in higher dimensions. In both cases one should use a more effi- cient simulation method, like the "go with the winner" strategy introduced by Grassberger [36 38 39]. It would be particularly interesting to study the expected crossover of $\pi_{t n}$, due to the proliferation of clusters, at and above the upper critical dimension [39]. It should be easier to do this for directed percolation, where $d_{\mathrm{c}}=4$, than for isotropic percolation, where $D_{\mathrm{c}}=6$.

The present work can be also extended by considering the crossing probability on same-spin clusters in strongly anisotropic spin systems at equilibrium, like the three-dimensional uniaxial ANNNI model at its Lifshitz point, for which the critical parameters are now accurately known [63].

\section{References}

1. R. P. Langlands, C. Pichet, P. Pouliot, Y. Saint-Aubin, J. Stat. Phys. 67, 553 (1992).

2. J. L. Cardy, J. Phys. A 25, L201 (1992).

3. R. M. Ziff, J. Phys. A 28, 1249 (1995).

4. R. M. Ziff, J. Phys. A 28, 6479 (1995).

5. H. T. Pinson, J. Stat. Phys. 75, 1167 (1994).

6. G. M. T. Watts, J. Phys. A 29, L363 (1996).

7. P. Kleban, Physica A 281, 242 (2000).

8. J. L. Cardy, Phys. Rev. Lett. 84, 3507 (2000).

9. R. P. Langlands, P. Pouliot, Y. Saint-Aubin, Bull. AMS 30, 1 (1994).

10. U. Gropengiesser, D. Stauffer, Physica A 210, 317 (1994).

11. D. Stauffer, J. Adler, A. Aharony, J. Phys. A 27, L475 (1994).

12. Y. Saint-Aubin, Physica A 221, 41 (1995).

13. C.-K. Hu, C.-Y. Lin, J.-A. Chen, Phys. Rev. Lett. 75, 193 (1995).

14. C.-K. Hu, J.-A. Chen, J. Phys. A 28, L73 (1995).

15. C.-K. Hu, C.-Y. Lin, J.-A. Chen, Physica A 221, 80 (1995).

16. J.-P. Hovi, A. Aharony, Phys. Rev. E 53, 235 (1996).

17. R. M. Ziff, Phys. Rev. E 54, 2547 (1996).

18. C.-K. Hu, C.-Y. Lin, Phys. Rev. Lett. 77, 8 (1996).

19. H.-P. Hsu, M.-C. Huang, K.-J. Ling, Phys. Rev. B 56, 10743 (1997).

20. M.-C. Huang, H.-P. Hsu, J. Phys. A 31, 3429 (1998).

21. C.-Y. Lin, C.-K. Hu, J.-A. Chen, J. Phys. A 31, L111 (1998).

22. C. D. Lorenz, R. M. Ziff, J. Phys. A 31, 8147 (1998).

23. M. Acharyya, D. Stauffer, Int. J. Mod. Phys. C 9, 643 (1998).

24. R. M. Ziff, C. D. Lorenz, P. Kleban, Physica A 266, 17 (1999).

25. S. Smirnov, C. R. Acad. Sci. Paris, Série I 333, 239 (2001).

26. O. Schramm, Elect. Comm. Probab. 6, 115 (2001).

27. R. P. Langlands, M.-A. Lewis, Y. Saint-Aubin, J. Stat. Phys. 98, 131 (2000).

28. E. Lapalme, Y. Saint-Aubin, J. Phys. A 34, 1825 (2001).

29. L.-P. Arguin, Y. Saint-Aubin, Phys. Lett. B 541, 384 (2002).

30. L.-P. Arguin, J. Stat. Phys., 109, 301 (2002).

31. C.-K. Hu, C.-Y. Lin, Phys. Rev. Lett. 77, 8 (1996).

32. P. Sen, Int. J. Mod. Phys. C 7, 603 (1996).

33. P. Sen, Int. J. Mod. Phys. C 8, 229 (1997).

34. L. N. Shchur, S. S. Kosyakov, Int. J. Mod. Phys. C 8, 473 (1997).

35. L. N. Shchur, S. S. Kosyakov, Nucl. Phys. B (Proc. Suppl.) 63AC, 664 (1998).

36. P. Grassberger, W. Nadler, talk presented at Heræus Summer School Chemnitz (2000), cond-mat/0010265

37. C.-K. Hu, J.-A. Chen, N. Sh. Izmailian, P. Kleban, Comp. Phys. Commun. 126, 77 (2000).

38. P. Grassberger, Comp. Phys. Commun. 147, 64 (2002).

39. L. N. Shchur L N, T. Rostunov, Pis'ma v ZhETF 76, 553 (2002).

40. M. Aizenman, Nucl. Phys. B 485, 551 (1997). 
41. M. Aizenman, Mathematics of Muliscale Materials, The IMA Volumes in Mathematics and its Applications, Vol. 8, edited by K. M. Golden, G. R. Grimmet, R. D. James, G. W. Milton and P. N. Sen (Springer Verlag, Heidelberg, 1998), p. 1.

42. J. L. Cardy, J. Phys. A 31, L105 (1998).

43. M. Aizenman, B. Duplantier, A. Aharony, Phys. Rev. Lett. 83, 1359 (1999).

44. J. L. Cardy, J. Phys. A 35, L565 (2002).

45. J. L. Cardy, Lectures on Conformal Invariance and Percolation, Bunkyo-ku, Tokyo (2001), math-ph/0103018

46. P. W. Kasteleyn, C. M. Fortuin, J. Phys. Soc. Japan (Suppl.) 26, 11 (1969).

47. P. W. Kasteleyn, C. M. Fortuin, Physica 57, 536 (1972).

48. L. Turban, Europhys. Lett. 60, 86 (2002).

49. L. Turban, J. Phys. A . 36, 3995 (2003).

50. K. Binder K., J.-S. Wang, J. Stat. Phys. 55, 87 (1989).

51. S. R. Broadbent, J. M. Hammersley, Proc. Camb. Philos. Soc. 53, 629 (1957)
52. W. Kinzel, Percolation Structures and Processes, Ann. Israel Phys. Soc. Vol. 5, edited by G. Deutscher, R. Zallen and J. Adler (Israel Physical Society, Haifa, 1983), p. 425.

53. H. Hinrichsen, Adv. Phys. 49, 815 (2000).

54. A. Hucht, J. Phys. A 35, L481 (2002).

55. Following a suggestion of the referee, the aspect ratio $r$ is defined here as $t / L^{z}$, which is the inverse of the definition previously used in [48] and [49]. With this more natural definition $r$ becomes a rescaled time of survival. The non-universal constant $c$ is also changed into its inverse.

56. T. E. Harris, Ann. Prob. 2, 969 (1974).

57. I. Jensen, J. Phys. A 32, 5233 (1999).

58. B. Derrida, J. Vannimenus, J. Physique (Lettres) 41, L473 (1980).

59. H. W. Watson, F. Galton, J. Anthropol. Inst. Great Britain and Ireland 4, 138 (1874).

60. H.-M. Bröker, P. Grassberger, Physica A 267, 453 (1999).

61. H.-K. Janssen, J. Stat. Phys. 103, 801 (2001).

62. A. Aharony, Y. Gefen, A. Kapitulnik, J. Phys. A 17, L197 (1984).

63. M. Pleimling, M. Henkel, Phys. Rev. Lett. 87, 125702 (2001). 